Dr Villamizar. Yes. There was only 1 study that reported very small numbers, like 10 in 1 group that underwent completion lobectomy after the lymph node was found positive, and the other was 5 that just had completion segmentectomy, probably because the patient could not tolerate a lobectomy or they thought that it was not appropriate, and the results were equivalent.
That being said, recently, Dr Hattori published a paper that even when the tumor is $2 \mathrm{~cm}$ but is solid, then lobectomy is better in terms of overall survival and locoregional recurrence than segmentectomy. So there are a lot of nuances that we need to pay attention to when deciding one surgery versus the other.
See Article page 2469.

\section{Commentary: How surprising}

\author{
Daniel J. Boffa, MD, and Michelle Salazar, MD
}

A perception exists within the thoracic community that segmentectomy represents an inferior lung cancer operation compared with lobectomy in the setting of nodal metastases. Among those beholden to this belief, the discovery of clinically occult ("surprise") nodal metastases during or after a planned segmentectomy would trigger the performance of a completion lobectomy (potentially as a second procedure). Razi and colleagues ${ }^{1}$ attempted to address the need for completion lobectomy by examining the outcome of clinical stage I patients who were upstaged from $\mathrm{cN} 0 \rightarrow \mathrm{pN} 1 / 2$ by segmentectomy or lobectomy. This is a clever approach to a relevant clinical question.

In theory, there are 2 ways that lobectomy could deliver superior locoregional control over segmentectomy: (1) more complete removal of tumor in parenchyma or (2) more complete removal of tumor in nodes. In terms of parenchymal clearance, the positive margin rate was approximately 3 percentage points greater in the segmentectomy cohort, compared with lobectomy. The study does not address skip lesions or satellite nodules (embedded in those upstaged to T3). Furthermore, the distance between tumor and the stapled parenchymal margin (which may have prognostic relevance) was unknown.

\footnotetext{
From Thoracic Surgery, Yale School of Medicine, New Haven, Conn.

Disclosures: Epic Sciences runs assays for free for Dr Boffa, unrelated to this work. Dr Salazar has nothing to disclose with regard to commercial support.

Received for publication Nov 29, 2019; revisions received Nov 29, 2019; accepted for publication Nov 30, 2019; available ahead of print Feb 4, 2020.

Address for reprints: Daniel J. Boffa, MD, P. O. Box 208062, New Haven, CT 06520 (E-mail: Daniel.boffa@yale.edu).

J Thorac Cardiovasc Surg 2020;159:2483-4 0022-5223/ $\$ 36.00$

Copyright (c) 2020 Published by Elsevier Inc. on behalf of The American Association for Thoracic Surgery

https://doi.org/10.1016/j.jtcvs.2019.11.118
}

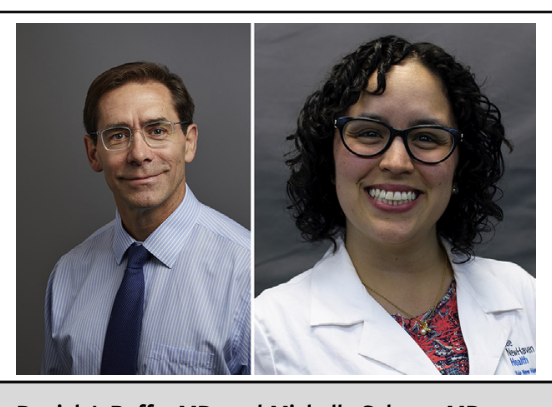

Daniel J. Boffa, MD, and Michelle Salazar, MD

CENTRAL MESSAGE

Overall survival appears to be similar when clinically nodenegative patients were surgically upstaged to node positive by lobectomy, or segmentectomy.

In terms of nodal clearance, there are a couple of signals worth mentioning. To start, nodal upstaging was more common in the lobectomy cohort than the segmentectomy cohort for both the $\mathrm{cN} 0 \rightarrow \mathrm{pN} 1(6.7 \%$ vs $2.5 \%)$ as well as the $\mathrm{cNO} \rightarrow \mathrm{pN} 2(3.9 \%$ vs $2.4 \%)$, which could reflect inferior lymph node evaluation during segmentectomy. However, this could also reflect a different risk for nodal metastases among "segmentectomy-amenable" (perhaps more peripheral) and "lobectomy-requiring" lung cancers. Within the cohort of upstaged patients, the patients who underwent lobectomy had more lymph nodes removed (eg, among those ending up pN1, 10.76 nodes vs 8.45 nodes in segmentectomy, $P<.001)$. The number of positive nodes in each cohort might be more reflective of risk for disease that was left behind. Although positive nodes were not examined in the study by Razi and colleagues, our team performed a cursory examination in the National Cancer Database attempting to mirror their population. Among clinical stage I lung cancer patients in the National Cancer Database upstaged from $\mathrm{cN} 0 \rightarrow \mathrm{pN} 1$, we found that 
$>1$ positive node was identified in $33 \%$ of patients who underwent segmentectomy and $41 \%$ of patients who underwent lobectomy. To be clear, this was unadjusted, and likely a somewhat-different patient population than that of Razi and colleagues, but is a signal worth pursuing. Combined, these findings (relating to margins and nodes) to some degree validate theoretical concerns that segmentectomy is an inferior cancer operation in this setting. And yet, the overall survival appears similar after lobectomy and segmentectomy. Why? It is unlikely that chemotherapy use is overcoming some oncologic disadvantage, because survival appeared similar after segmentectomy and lobectomy, in the presence and absence of adjuvant chemotherapy. Perhaps any survival disadvantage of segmentectomy as a cancer operation was balanced by an overall survival advantage of taking less lung parenchyma. Perhaps the patients who underwent segmentectomy had a more favorable prognosis, irrespective of procedure performed (ie, more favorable tumor biology).

All things considered, this study feels like a potentially practice-changing study. The question is how. The answer may relate to "how surprising" the nodal metastases were in each cohort. There is some evidence supporting an association between nodal disease burden and prognosis (ie, number of involved nodes, gross vs microscopic involvement). ${ }^{2-4}$ If nodal cancer burden was indeed prognostic, then the manner in which nodes were discovered could create survival bias in the cohorts. Consider the following scenarios:

Unfortunately, nothing is known about the intraoperative decision making, which limits our ability to apply these findings (we don't have enough information to emulate what was done).

- These data do not support (or reject) the conclusion that segmentectomy and lobectomy are equivalent for all node-positive lung cancers (in fairness, not an assertion of Razi and colleagues).

- However, the data do indicate that when board-certified thoracic surgeons believe a segmentectomy for clinical stage I lung cancer is appropriate (including his or her intraoperative analyses/judgment), for what ultimately

\begin{tabular}{ll}
\hline \multicolumn{1}{c}{ Scenario } & "Surprise factor" \\
\hline $\begin{array}{l}\text { All lobectomies and segmentectomies took } \\
\text { place as planned without any reaction to }\end{array}$ & Average in both \\
intraoperative findings. & \\
Grossly abnormal nodes were analyzed by & $\begin{array}{c}\text { More surprising in } \\
\text { segmentectomy }\end{array}$ \\
frozen section. All planned & patients \\
segmentectomies with positive nodes were \\
converted to lobectomy. Nodal metastases \\
in segmentectomy patients occurred in \\
grossly normal nodes, not evaluated by \\
frozen section. \\
$\begin{array}{l}\text { Frozen section was performed on all nodes. } \\
\text { Segmentectomies only performed in }\end{array}$ \\
$\begin{array}{l}\text { patients in which all nodes were negative } \\
\text { for cancer on frozen section. Nodal }\end{array}$ \\
$\begin{array}{l}\text { metastases in segmentectomy patients } \\
\text { were microscopic metastases (missed on }\end{array}$ \\
frozen section). \\
\hline
\end{tabular}

proves to be a node-positive lung cancer, similar overall survival to lobectomy can be achieved.

As with any retrospective study in which patient selection took place that is not characterized by the available data, the findings illustrate what is possible, not necessarily what is probable. The data support continuation of the selective segmentectomy practice of surgeons contributing to the Society of Thoracic Surgeons database. Efforts to characterize decision making of the surgeons from the Society of Thoracic Surgeons in this setting could increase the generalizability and overall impact of these findings.

\section{References}

1. Razi SS, Nguyen D, Villamizar N. Lobectomy does not confer survival advantage over segmentectomy for non-small cell lung cancer with unsuspected nodal disease. J Thorac Cardiovasc Surg. 2020;159:2469-83.e4.

2. Detterbeck F. What to do with "surprise" N2?: intraoperative management of patients with non-small cell lung cancer. J Thorac Oncol. 2008;3:289-302.

3. Fiorelli A, Sagan D, Mackiewicz L, Cagini L, Scarnecchia E, Chiodini P, et al. Incidence, risk factors, and analysis of survival of unexpected N2 disease in stage I non-small cell lung cancer. Thorac Cardiovasc Surg. 2015;63:558-67.

4. Marchevsky AM, Gupta R, Kusuanco D, Mirocha J, McKenna RJ Jr. The presence of isolated tumor cells and micrometastases in the intrathoracic lymph nodes of patients with lung cancer is not associated with decreased survival. Hum Pathol. 2010;41:1536-43. 